\title{
ISO 11404
}

National Cancer Institute

\section{Source}

National Cancer Institute. ISO 11404. NCI Thesaurus. Code C81892.

A standard that specifies the nomenclature and shared semantics for a collection of data types commonly occurring in programming languages and software interfaces. 